\title{
Non-destructive Characterization of Dielectric - Semiconductor Interfaces by Second Harmonic Generation
}

I. Ionica ${ }^{\mathrm{a}}$, D. Damianos ${ }^{\mathrm{a}}$, A. Kaminski ${ }^{\mathrm{a}}$, G. Vitrant ${ }^{\mathrm{a}}$, D. Blanc-Pélissier ${ }^{\mathrm{b}}$, J. Changala $^{\mathrm{c}}$, M. Kryger ${ }^{\mathrm{c}}$, C. Barbos ${ }^{\mathrm{b}}$, and S. Cristoloveanu ${ }^{\mathrm{a}}$

${ }^{a}$ IMEP-LAHC, Minatec, Grenoble-INP, Univ. de Grenoble Alpes, CNRS-UMR 5130, 3 Parvis Louis Néel - CS 50257, F-38016 Grenoble, France

${ }^{\mathrm{b}}$ INL - UMR 5270, INSA de Lyon, 7 avenue Jean Capelle, 69621 Villeurbanne, France ${ }^{\mathrm{c}}$ FemtoMetrix, 1850 East Saint Andrew Place, Santa Ana, CA 92705, USA

\begin{abstract}
Here we present the characterization of dielectric - semiconductor interfaces using optical second harmonic generation (SHG). The technique is contactless, which makes it useful for non-destructive monitoring of ultra-thin dielectrics. The generated second harmonic is sensitive to material parameters and charges near interfaces. The signal exhibits time dependence due to charging of traps with carriers generated by the incident laser during the measurement. Quantitative models are applied to the time dependent $\mathrm{SHG}$ signals in the $\mathrm{Al}_{2} \mathrm{O}_{3}$ stacks, which provide trapping kinetics information, and thus $\mathrm{Al}_{2} \mathrm{O}_{3}$ film quality. $\mathrm{Al}_{2} \mathrm{O}_{3}$ films are commonly used for passivating $\mathrm{Si}$ in photovoltaic stacks. To further demonstrate that time dependent behavior is due to changes in the electric field across the semiconductor/dielectric interface, additional systems are presented for comparison: $\mathrm{SiN}_{\mathrm{x}}$ on silicon, passivated and non-passivated silicon-on-insulator substrates. Results confirm the value of SHG as a quality control method for multiple thin film dielectrics.
\end{abstract}

\section{Introduction}

High quality interfaces between dielectrics and semiconductors are mandatory not only for microelectronic devices, but also for other applications like photovoltaics where the electrical quality of the passivation layers is crucial in order to obtain high yield solar cells (1). The challenge in producing excellent surface quality is not only in the fabrication process; it also requires effective characterization techniques.

One of the key characterization needs of semiconductor devices is the non-destructive measurement of electrical properties of the interfaces between dielectric materials and semiconductors. For example, the ITRS 2013 (2) mentions that the measurement of local interfacial electrical properties are among the difficult challenges for metrology. In this context, a characterization tool based on optical second harmonic generation (SHG) (3) fulfills critical metrology requirements: it is contactless, non-destructive, sensitive and can provide a high spatial resolution.

In this paper, after a brief overview of the principles of $\mathrm{SHG}$, we review its use for dielectric material characterization and provide results on $\mathrm{Al}_{2} \mathrm{O}_{3} / \mathrm{Si}$ stacks. SHG measurements on $\mathrm{Al}_{2} \mathrm{O}_{3}$ on silicon are compared with results obtained on $\mathrm{SiN}_{\mathrm{x}}$ on silicon and on passivated and non-passivated silicon-on-insulator (SOI) wafers. 


\section{Second Harmonic Generation}

\section{$\underline{\text { Principle }}$}

Second Harmonic Generation is a non-linear optical effect, related to the polarization of materials under the influence of a high intensity light. A monochromatic light of angular frequency $\omega$ (wavelength $\lambda$ ) and electric field amplitude $\vec{E}(\omega)$ incident on a material induces a polarization, $\vec{P}$. The linear polarization is given by the product of the incident electric field with the first order susceptibility $\chi^{(1)}$ :

$$
\vec{P}^{(1)}(\omega)=\chi^{(1)} \cdot \vec{E}(\omega)
$$

In the most general case, the polarization contains not only this linear term but also terms involving higher order susceptibility tensors (4):

$$
\vec{P}=\vec{P}^{(1)}+\vec{P}^{(2)}+\vec{P}^{(3)}+\ldots=\chi^{(1)} \cdot \vec{E}(\omega)+\vec{\chi}^{(2)} \cdot \vec{E}(\omega) \cdot \vec{E}(\omega)+\vec{\chi}^{(3)} \cdot \vec{E}(\omega) \cdot \vec{E}(\omega) \cdot \vec{E}(\omega)+\ldots
$$

Second harmonic generation is related to the second order term in equation (2), which has a characteristic angular frequency of $2 \cdot \omega$ (wavelength $\lambda / 2$ ). Figure 1 shows the principle of SHG.

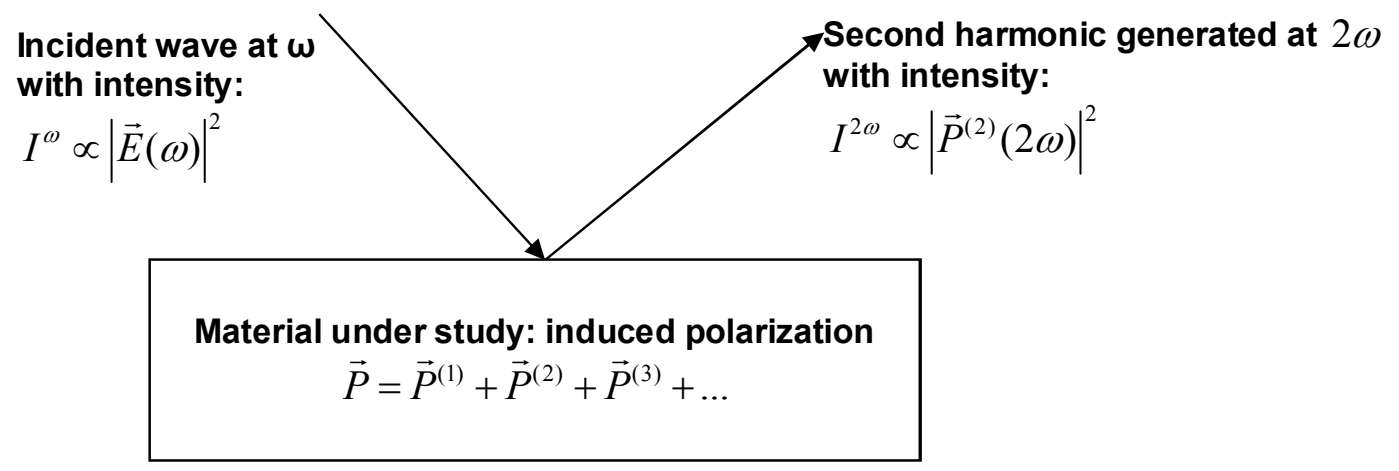

Figure 1. Principle of SHG.

Additionally, in presence of an internal electric field inside the material, $E_{\text {int }}$, a supplementary contribution is added to the second order polarization; its associated SHG signal is called EFISH (electric field induced second harmonic). The EFISH term is related to the $3^{\text {rd }}$ order susceptibility tensor $\vec{\chi}^{(3)}$. Consequently, the full second harmonic intensity, given by the square modulus of the total $2^{\text {nd }}$ order polarization, is written as:

$$
I^{2 \omega} \sim\left|\vec{\chi}^{(2)}+\vec{\chi}^{(3)} \cdot \vec{E}_{\text {int }}\right|^{2} \cdot|\vec{E}(\omega)|^{4}
$$

For centro-symetric materials such as silicon, silicon dioxide etc., the second order susceptibility tensor $\vec{\chi}^{(2)}$ of the bulk is zero. However, any symmetry-breaking (for example at the interfaces between two materials) induces non-zero $2^{\text {nd }}$ order susceptibility $\vec{\chi}_{\text {interface }}^{(2)}$ and its associated SHG signal reflects the interface quality. This second order susceptibility tensor contains 27 components, but thanks to axes symmetry for most of the interfaces used in microelectronics (for example $\mathrm{Si} / \mathrm{SiO}_{2}$ ), only 5 components are non-zero, and only 3 of them are independent (5).

The term associated with the electric field in equation (3) is the most relevant when studying material stacks such as dielectrics on silicon, since $E_{\text {int }}$ is related to all charges close to the interfaces between materials (i.e. fixed oxide charges, interface states, 
charged defects, space charge region in the semiconductor...). Furthermore, under the incident laser influence, electron-hole pairs can be generated in the semiconductor and they can be injected into defects/trapped at defect sites, thus modulating the electric field at the interface. Therefore, $E_{\text {int }}$ can be time-dependent due to trapping phenomena at the interface.

\section{$\underline{\text { Second Harmonic Generation for Dielectric Characterization }}$}

The variable parameters of referenced SHG experiments are: the wavelength, the power and the polarization of the incident beam, the polarization of the detector, the angle of incidence, the azimuthal angle of the sample. Each of these experimental parameters gives access to different kind of information concerning the material under study. For example, spectroscopic SHG (i.e. with various wavelengths), was used to identify interband resonances at silicon interfaces and different bonds contributions (6), or to determine the polarity of charges at $\mathrm{Al}_{2} \mathrm{O}_{3} / \mathrm{Si}$ interfaces (7).

The incident power of the laser impacts the generation of carriers in the semiconductor film or substrate and can activate multi-photon excitations, which influence charging effects of different types of traps in the stack. For example, for $\mathrm{SiO}_{2}$ on highly borondoped $\mathrm{Si}$, depending on the incident power of the laser, it was possible to induce trapping only in boron-induced defects in the oxide or in both boron-induced defects and oxygen traps on the top oxide surface (8).

The variation of the SHG signal with respect to the azimuthal angle (rotational angle of the sample around its surface normal) is associated to the symmetry of the substrate (9) and also to its roughness. For example, Dadap et al. showed a good correlation between the surface roughness during the first stages of native oxidation and the SHG response to azimuthal angle (10).

The couple input polarization - output polarization allows selecting specific components of the susceptibility tensors (11), (12), (13), and thus the different components can be determined.

Time-dependent SHG was used to characterize trapping - detrapping phenomena, in a variety of dielectric materials: $\mathrm{SiO}_{2} / \mathrm{Si}(8), \mathrm{Al}_{2} \mathrm{O}_{3}(14), \mathrm{ZrO}_{2}$ (15), and silicon-on-insulator (16).

The most intensive studies of $\mathrm{SHG}$ were performed on $\mathrm{SiO}_{2} / \mathrm{Si}$ interfaces, which is of importance to microelectronic devices. A review of the main results obtained with SHG (17) shows relevant information extracted for silicon dioxide on silicon: micro-roughness, strain effects, bonding, carrier injection.

SHG proved to be also of high interest for high-k dielectric materials, especially $\mathrm{Al}_{2} \mathrm{O}_{3}$, bringing remarkable insights on:

- the importance of the thickness of the intermediate $\mathrm{SiO}_{2}$ layer (always present at the interface with $\mathrm{Si}$ ) on the charge polarity in the stack $\mathrm{Si} / \mathrm{SiO}{ }_{2} / \mathrm{Al}_{2} \mathrm{O}_{3}$ (observed by spectroscopic SHG, time-dependent SHG and SHG versus azimuthal angle) (7),

- the identification of a fixed negative charge in the $\mathrm{Al}_{2} \mathrm{O}_{3}$ layer that increases after annealing (observed by spectroscopic and time-dependent SHG) (14),

- "Optical roughness," a quantity that reflects the non-uniform distribution of the interfacial defects capable of charge trapping (observed using rotational angle SHG for ultra-thin high-k dielectric stacks) (18). 


\section{$\mathrm{Al}_{2} \mathrm{O}_{3}$ Characterization by SHG}

In modern microelectronics, there is strong interest in high-k materials to replace $\mathrm{SiO}_{2}$ as a gate dielectric in MOS structures, to increase transistor performance. $\mathrm{Al}_{2} \mathrm{O}_{3}$ is one of the dielectric materials used for III-V transistors (19). Additionally, $\mathrm{Al}_{2} \mathrm{O}_{3}$ gives excellent surface passivation of crystalline silicon, which is critical for the performance of devices such as light-emitting diodes, photodetectors and high-efficiency solar cells (20). The characterization of its interface with the semiconductor material on which it is deposited is in both cases critical.

\section{$\underline{\text { Sample Preparation }}$}

Substrates were $250 \mu \mathrm{m}$ thick float zone (FZ) p-type double side polished Si (100) and (111) with a resistivity of $0.8 \Omega . \mathrm{cm}$. Prior to deposition, the wafers were cleaned using a standard chemical process (Piranha $\left[\mathrm{H}_{2} \mathrm{O}_{2}, \mathrm{H}_{2} \mathrm{SO}_{4}\right]$ and diluted $\mathrm{HF}$ ). $\mathrm{Al}_{2} \mathrm{O}_{3}$ films with 15 $\mathrm{nm}$ thickness were grown by thermal atomic layer deposition (ALD) in an Ultratech Fiji F200 reactor on both sides of the substrates. Trimethylaluminium $\left(\mathrm{Al}\left(\mathrm{CH}_{3}\right)_{3}\right)$ and water $\left(\mathrm{H}_{2} \mathrm{O}\right)$ were used as reactants. The chuck and chamber temperature was set to $250^{\circ} \mathrm{C}$ during deposition. After as-deposited characterizations, the samples were annealed in the ALD chamber at $400^{\circ} \mathrm{C}$ for $10 \mathrm{~min}$. The annealing step is essential for photovoltaic applications to activate the negative surface charges and improve the surface passivation (21).

$\mathrm{Si}(100)$ and $\mathrm{Si}(111)$ wafers with $15 \mathrm{~nm}$ amorphous $\mathrm{Al}_{2} \mathrm{O}_{3}$ deposited on both sides were cut in half after deposition and first characterizations. One half was kept as reference and the other half was annealed. As-deposited $\mathrm{Al}_{2} \mathrm{O}_{3}$ layers grown by thermal-ALD provide a significant level of surface passivation of $\mathrm{Si}$ substrates mainly due to the reduction of the electrically active interface states by chemical bonding ( $\mathrm{Si}-\mathrm{O}-\mathrm{Al}$ ). However, further reduction of the surface recombination requires the activation of charges at the $\mathrm{Al}_{2} \mathrm{O}_{3} / \mathrm{Si}$ interface that provides a field-effect passivation. The negative charges attributed to interstitial oxygen and/or aluminum vacancies are activated by thermal annealing. Comparative characterization steps that will be described in the next sub-sections were used to evaluate the effect of this supplementary passivation.

For comparison, $70 \mathrm{~nm}$-thick $\mathrm{SiN}_{\mathrm{x}}: \mathrm{H}$ coatings with positive interface charges (attributed to specific silicon-nitrogen dangling bonds) were deposited on both sides of $\mathrm{p}$ type FZ Si substrates (resistivity $5 \Omega . \mathrm{cm}$ ) by plasma-assisted chemical vapor deposition (PECVD). $\mathrm{SiN}_{\mathrm{x}}: \mathrm{H}$ layers are commonly used as standard passivation anti-reflection coatings for silicon solar cells. However due to their positive charges, these layers are not suited to passivate highly doped $\mathrm{p}+$ region at the back-side of $\mathrm{p}$-type silicon cells. 


\section{Minority Carrier Lifetime Measurements}

Prior to SHG measurements, the surface passivation of silicon was evaluated after each technological step by measuring the effective minority carrier lifetime $\left(\tau_{e f f}\right)$. For a symmetrically passivated wafer with sufficiently low surface defect density, $\tau_{\text {eff }}$ can be expressed as a bulk lifetime $\left(\tau_{b u l k}\right)$ and a surface contribution $(2 S / e)$ :

$$
\frac{1}{\tau_{\text {eff }}}=\frac{1}{\tau_{\text {bulk }}}+\frac{2 S}{e}
$$

where $S$ is the surface recombination velocity and $e$ is the wafer thickness. For high resistivity $\mathrm{Si}$ substrates with high bulk lifetimes, $S$ can be directly related to $\tau_{\text {eff }}$ by $S=\frac{e}{2 \cdot \tau_{e f f}}$

Spatially resolved effective minority carrier lifetime mapping was performed by microwave photo-conductance decay (Semilab WC-2000 $\mu \mathrm{W}-\mathrm{PCD}$ ) on the passivated samples, before and after annealing. The equipment is using a laser source emitting at $904 \mathrm{~nm}$ with a short light pulse of $200 \mathrm{~ns}$ to create electron-hole pairs. The excess carriers modify the conductivity, which is monitored by microwave reflectivity as a function of time. The minority carrier lifetime is determined via the decay of the photoconductivity with time. The mapping step was set to $500 \mu \mathrm{m}$. Figure 2 shows the maps obtained on the $\mathrm{Al}_{2} \mathrm{O}_{3}$ coated $\mathrm{Si}(100)$ and on $\mathrm{Si}(111)$, as-deposited (Figure $2 \mathrm{a}$ and $\mathrm{b}$ ) and after annealing (Figure 2c). The averaged $\tau_{\text {eff }}$ value was $57 \mu \mathrm{s}$ for $\mathrm{Al}_{2} \mathrm{O}_{3} / \mathrm{Si}(100)$ and $81 \mu \mathrm{s}$ for $\mathrm{Al}_{2} \mathrm{O}_{3} / \mathrm{Si}(111)$ before annealing. After annealing, these values increased to $140 \mu \mathrm{s}$ and $250 \mu$ s respectively. The higher values of lifetime measured after annealing for each case confirm the reduction of surface recombination due to the activation of the negative charges at the interface $\mathrm{Al}_{2} \mathrm{O}_{3} / \mathrm{Si}$. Additionally, higher lifetime values are obtained when the alumina is deposited on $\mathrm{Si}(111)$ rather than on $\mathrm{Si}(100)$, suggesting a better passivation. The effect can be related to a higher quality substrate, since the $\operatorname{Si}(111)$ is known to produce better surfaces than $\mathrm{Si}(100)$. 
(a) $\mu \mathrm{W}$-PCD wafer map of as-deposited $\mathrm{Al}_{2} \mathrm{O}_{3}$ on $\mathrm{Si}(100)$. Shorter minority carrier lifetimes.

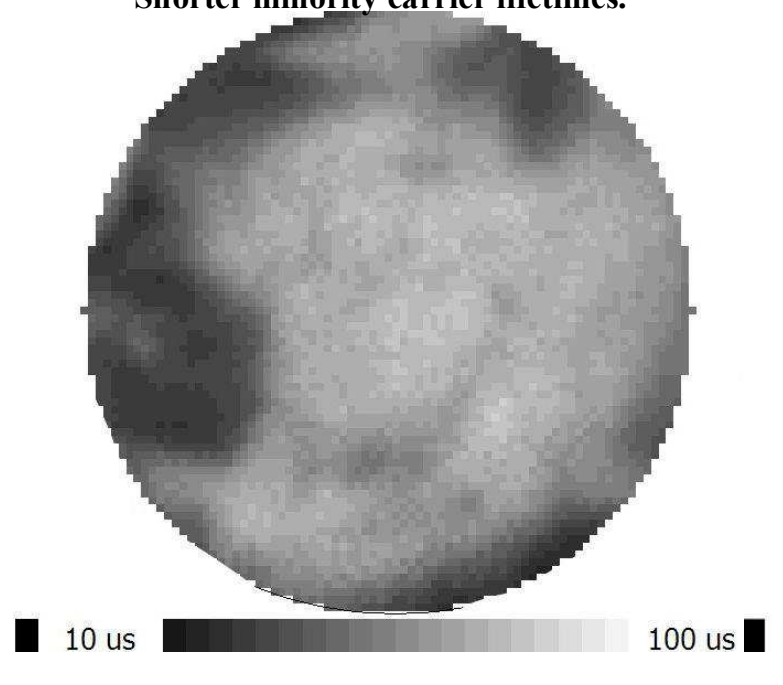

(b) $\quad \mu \mathrm{W}$-PCD wafer map of as-deposited $\mathrm{Al}_{2} \mathrm{O}_{3}$ on $\mathrm{Si}(111)$. Longer minority carrier lifetimes.

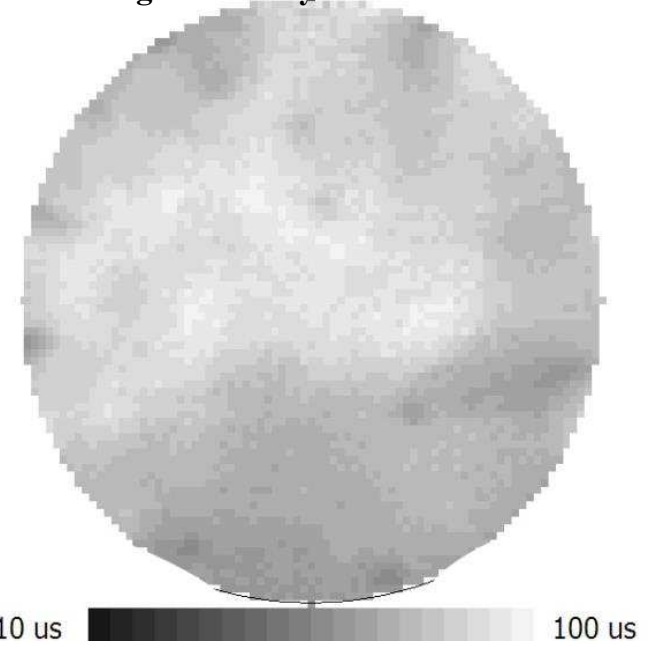

(c)

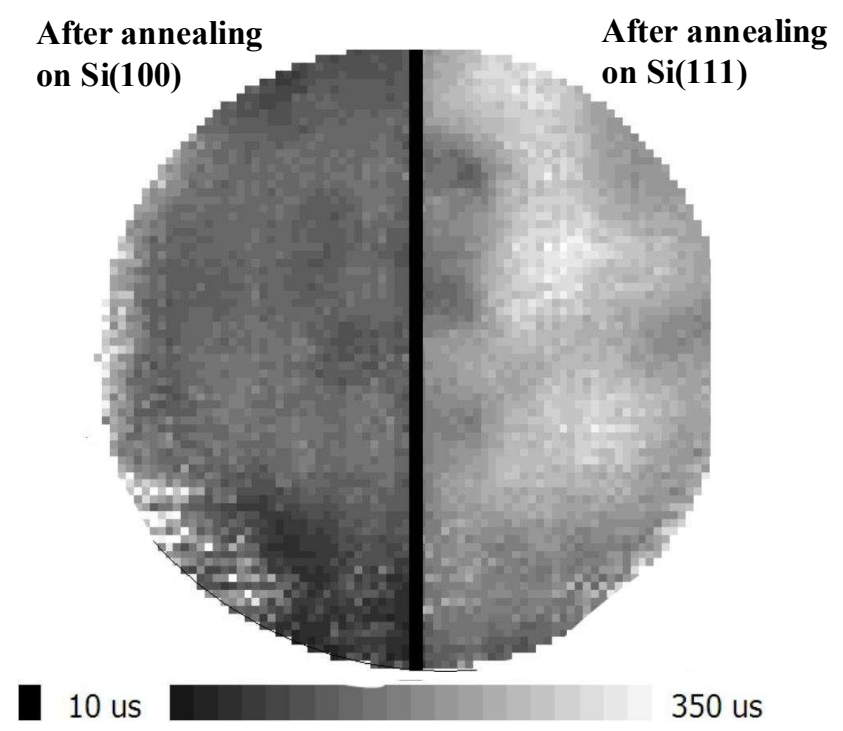

Figure 2: Minority carrier lifetime mapping on $\mathrm{Al}_{2} \mathrm{O}_{3}$ coated $\mathrm{Si}(100)$ (a) and $\mathrm{Si}(111)$ (b). The samples were then cleaved in half and thermally annealed. The minority carrier lifetime maps of the annealed half of each sample are shown in (c), demonstrating longer lifetimes post-anneal.

\section{$\underline{\text { SHG Experimental Set-up }}$}

SHG experimental results were obtained on the Harmonic F1x ${ }^{\circledR}$ optical SHG wafer inspection system from FemtoMetrix (22). A $780 \mathrm{~nm}$ wavelength laser delivers femtosecond pulses to the sample. The laser power is $\sim 380 \mathrm{~mW}$. The incident polarization is controlled by a half wave plate. The second harmonic generated in the sample at the double incident frequency (390 $\mathrm{nm}$ wavelength) goes through a filter and a rotating polarizer which allows measuring different output polarization states of the SHG 
beam. The SHG beam is detected by a photomultiplier coupled with a photon counter. The experimental parameters that can be modified are the azimuthal angle of the sample, the angle of incidence of the incident beam, and the polarization states of the incident and the second harmonic beam. The sample is placed on an automated stage stack which allows mapping. Wafers up to $300 \mathrm{~mm}$ diameter can be tested.

\section{Surface Mapping SHG}

The same samples measured for the carrier lifetime mapping were also used for SHG maps. The angle of incidence was set at $45^{\circ}$. P-polarizations were used for both incident and detected beams. Figure 3 was obtained on $\mathrm{Al}_{2} \mathrm{O}_{3}$ deposited on $\mathrm{Si}(100)$, while in Figure 4 the substrate was $\mathrm{Si}(111)$. After annealing the SHG signal is stronger by a factor of 2 roughly, for both types of substrates. Note that the SHG values measured on $\mathrm{Si}(111)$ were higher. A quick comparison with the minority carrier lifetime maps in Figure 2 suggests that a higher lifetime corresponds to a stronger SHG signal. In order to check whether this correlation is correct, we also tested $\mathrm{SiN}_{\mathrm{x}}$ standard and $\mathrm{SiN}_{\mathrm{x}} \mathrm{Si}$-rich samples. Figure 5 shows the minority carrier lifetime and the SHG signal versus time for both types of samples. The standard $\mathrm{SiN}_{\mathrm{x}}$ has a lower lifetime (averaged $\tau_{\text {eff }} \sim 265 \mu \mathrm{s}$ ) but a higher SHG signal than the Si-rich $\operatorname{SiN}_{\mathrm{x}}$ (averaged $\tau_{\text {eff }} \sim 420 \mu \mathrm{s}$ ) which indicates that carrier lifetime and SHG intensity are not directly linked. However, a direct comparison of the SHG values should not be done since the optical properties of the two materials are very different: Si-rich $\mathrm{SiN}_{\mathrm{x}}$ is known to absorb more than the standard $\mathrm{SiN}_{\mathrm{x}}$, both at the harmonic and second harmonic frequency.

Generally, care must be taken when comparing SHG on different materials, so the color maps are only relevant if the material studied is the same. Otherwise, the SHG should be analyzed versus time, as shown in the next section.

(a)

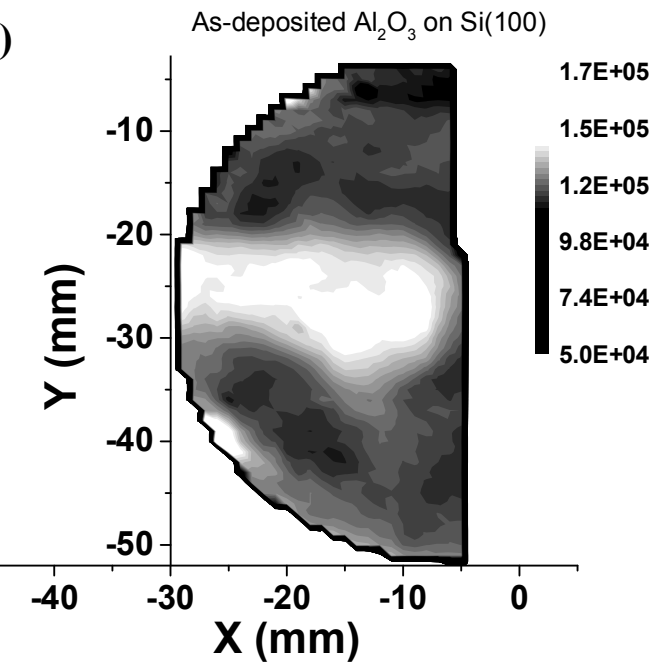

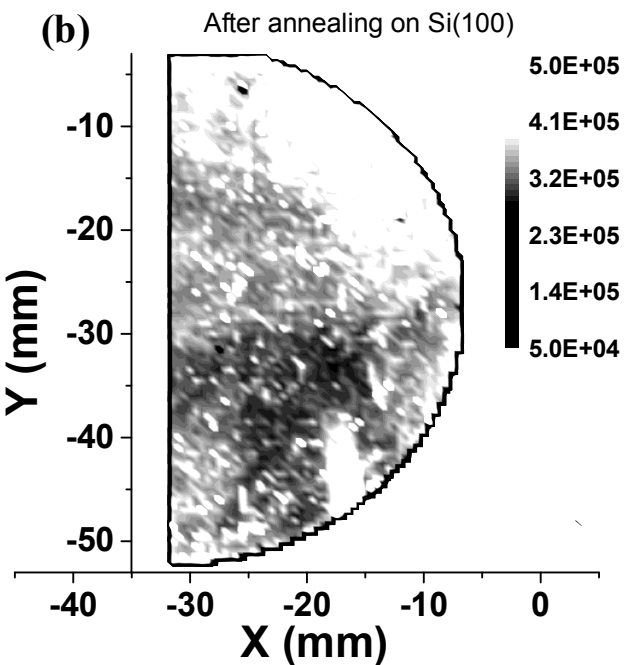

Figure 3: SHG maps on $\mathrm{Al}_{2} \mathrm{O}_{3}$ deposited on $\mathrm{Si}$ (100): (a) as-deposited and (b) after annealing. The lateral step of the measurements is of $1 \mathrm{~mm}$. The black color represents the same value for both experiments. Nevertheless the white color corresponds to a much lower SHG signal in (a) than in (b). 

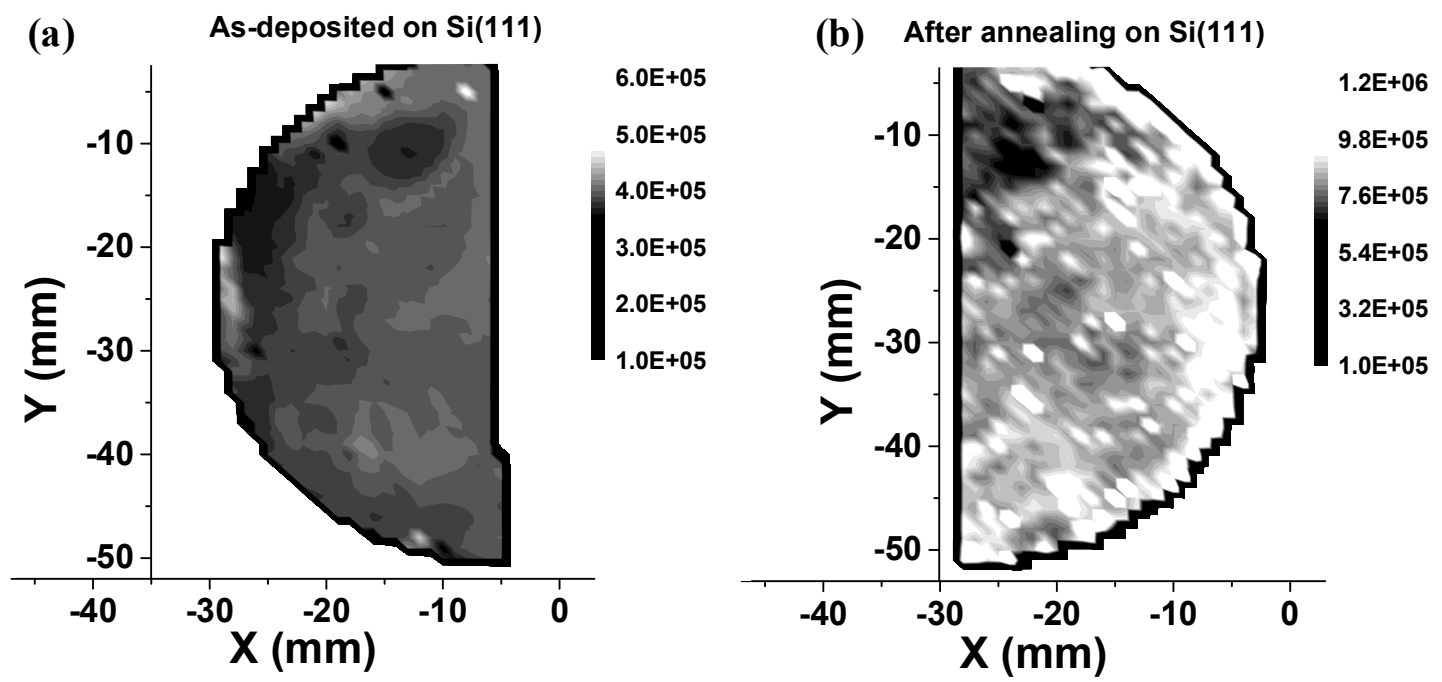

Figure 4: SHG maps on $\mathrm{Al}_{2} \mathrm{O}_{3}$ deposited on $\mathrm{Si}$ (111): (a) as-deposited and (b) after annealing. The lateral step of the measurements is of $1 \mathrm{~mm}$. The minimum value (in black) is the same for both experiments. However, the maximum value (in white color) corresponds to a much lower SHG signal in (a) than in (b).

(a)

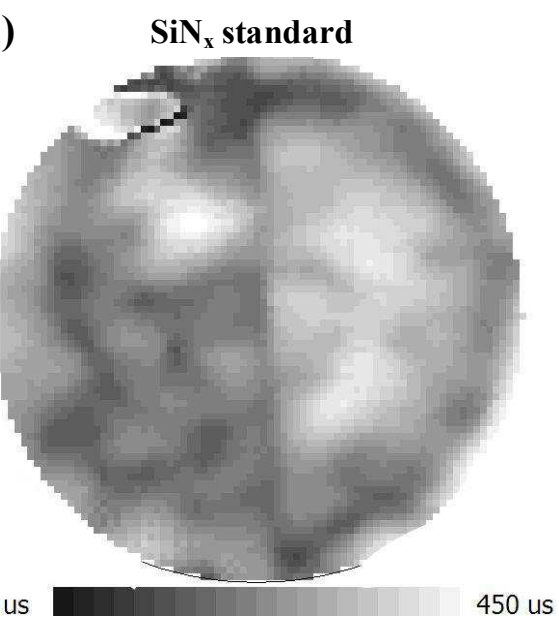

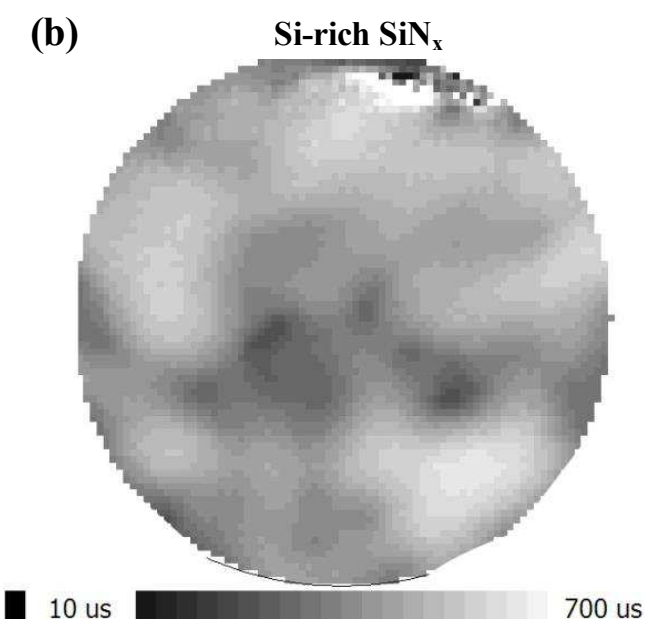

(c)

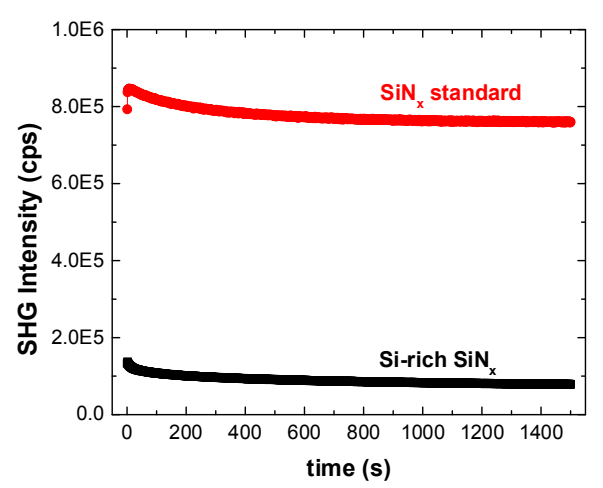

Figure 5: Minority carrier lifetime $(\mathrm{a}, \mathrm{b})$ and time-dependent SHG (c) for two samples passivated with $\mathrm{SiN}_{\mathrm{x}}$ standard (a) and silicon-rich (b). 


\section{Time-dependent SHG}

In this section we use time-dependent SHG to develop a deeper understanding of the material properties. The only parameter that could depend on time in equation (3) is the electric field, which can have a non-zero initial value related to charges present in the materials or at the interfaces and a time-dependent part which represents trapping of carriers generated by the laser exposure during the experiment. The SHG intensity versus time can be written as:

$$
I^{2 \omega}(t) \sim\left|\vec{\chi}^{(2)}+\vec{\chi}^{(3)} \cdot\left(E_{0}+E(t)\right)\right|^{2} \cdot\left|I^{\omega}\right|^{2}
$$

The interest of time-dependent SHG appears mainly when studying stacks in which charging effects manifest themselves. Exponential behavior associated with different trapping phenomena have been identified in thin oxide dielectrics on Si (8), (15).

Figure 6 shows the time-dependent SHG signal obtained on the samples studied in Figure 3 and Figure 4 . In all cases a fast increase of the signal is followed by a much slower variation. For both substrates, the SHG increases after annealing, in agreement with the activation of negative charges at the interface, during thermal treatment (23). If we compare these curves with the ones shown in Figure $5 \mathrm{c}$ for $\mathrm{SiN}_{\mathrm{x}}$, the time-response is obviously different. The charges in standard $\mathrm{SiN}_{\mathrm{x}}$ are known to be positive due to specific silicon-nitrogen dangling bonds (24); the electrons generated by the incident laser that are injected in the dielectrics will reduce the effective positive charge and therefore reduce the electric field and the EFISH.

Mihaychuk et al. proved that the SHG intensity is proportional to the square of the surface charge density in $\mathrm{SiO}_{2} / \mathrm{Si}$ interfaces (25) and this relationship was also used for $\mathrm{Al}_{2} \mathrm{O}_{3}$ (7), (14). Consequently, the initial value $I^{2 \omega}(t=0)$ is related to the fixed charges in the dielectric. Calibration with another measurement technique like capacitance measurements on MIS structures lead to an estimation of the fixed charge density (18).
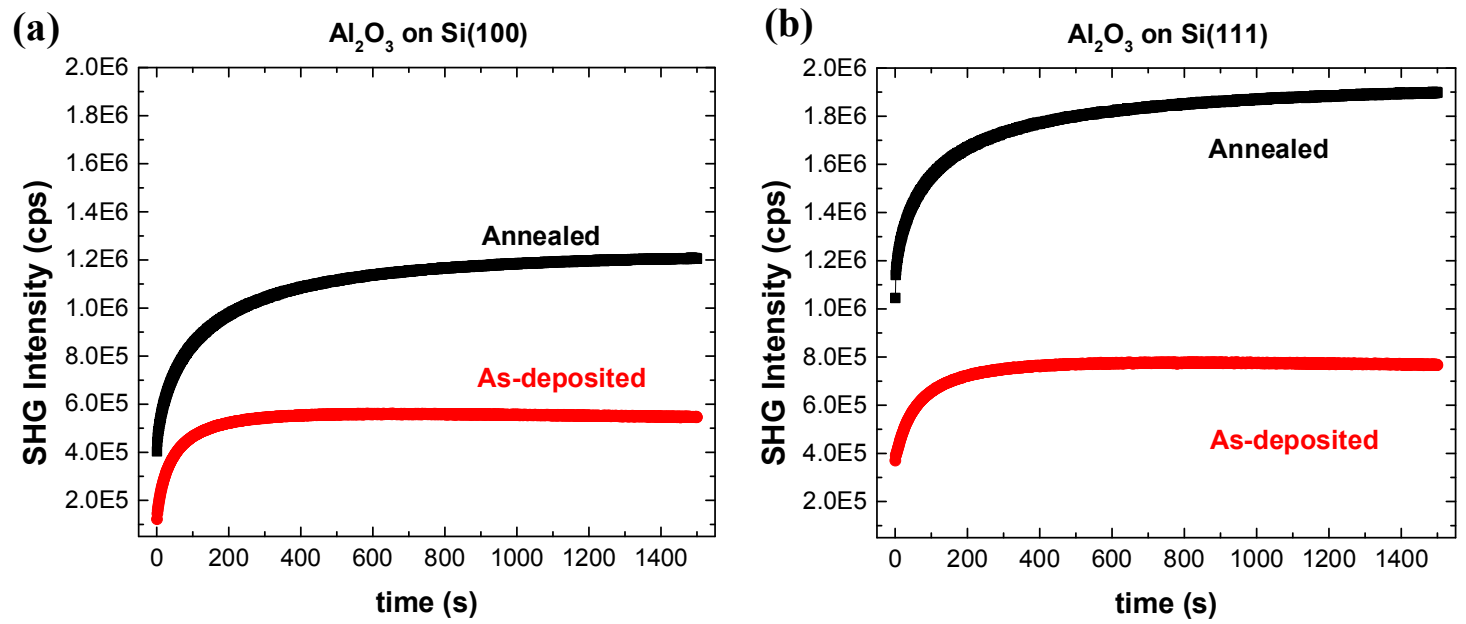

Figure 6: Time-dependent $\mathrm{SH}$ generated signal from the $\mathrm{Al}_{2} \mathrm{O}_{3}$ on $\mathrm{Si}(100)$ (a) and on $\operatorname{Si}(111)(b)$. The same vertical scales were used. 
In order to evidence charging phenomena, we plotted the square-root of the SHG intensity versus time (Figure 7). Based on equation (5), this graph is an image of the time evolution of the electric field, due to charging phenomena produced by the laser illumination. The curves were fitted using two exponentials:

$$
y=y_{0}+A_{1} \cdot\left(1-e^{-\frac{x}{\tau_{1}}}\right)+A_{2} \cdot\left(1-e^{-\frac{x}{\tau_{2}}}\right)
$$

A model with two exponentials was already used to explain charging effects in $\mathrm{SiO}_{2}$ deposited on highly doped $\mathrm{Si}(8)$. In $\mathrm{Al}_{2} \mathrm{O}_{3}$, Gielis et al. (14) used a fitting model with 3 exponentials. The charging occurs thanks to the electrons of the silicon substrate that are injected at the interface, inside the bulk of the dielectric or at the ambient surface (18), (26). The thickness of $\mathrm{Al}_{2} \mathrm{O}_{3}$ in our samples is $15 \mathrm{~nm}$, which makes charging at the surface highly unlikely. The defects responsible for charging in the stack can be related to interstitial oxygen or aluminum vacancies, both producing defect states in the $\mathrm{Al}_{2} \mathrm{O}_{3}$ below mid-gap that can be negatively charged (27). The monotonic increase in the SHG signal suggests that the injected charges have the same sign as the fixed charges, known to be negative here (7) which agrees with the laser-induced electron injection from the Si substrate. It should be kept in mind that the native $\mathrm{SiO}_{2}$ layer (thickness $<1 \mathrm{~nm}$ ) present at the $\mathrm{Al}_{2} \mathrm{O}_{3} / \mathrm{Si}$ interface may play a role in the charge transfer (7).

The table below shows the time constants extracted from our fits. Annealing had the effect of increasing the time constants. The larger time constants associated with the slow increase of the signal can be explained by the saturation of the trapping sites in $\mathrm{Al}_{2} \mathrm{O}_{3}$ (7).
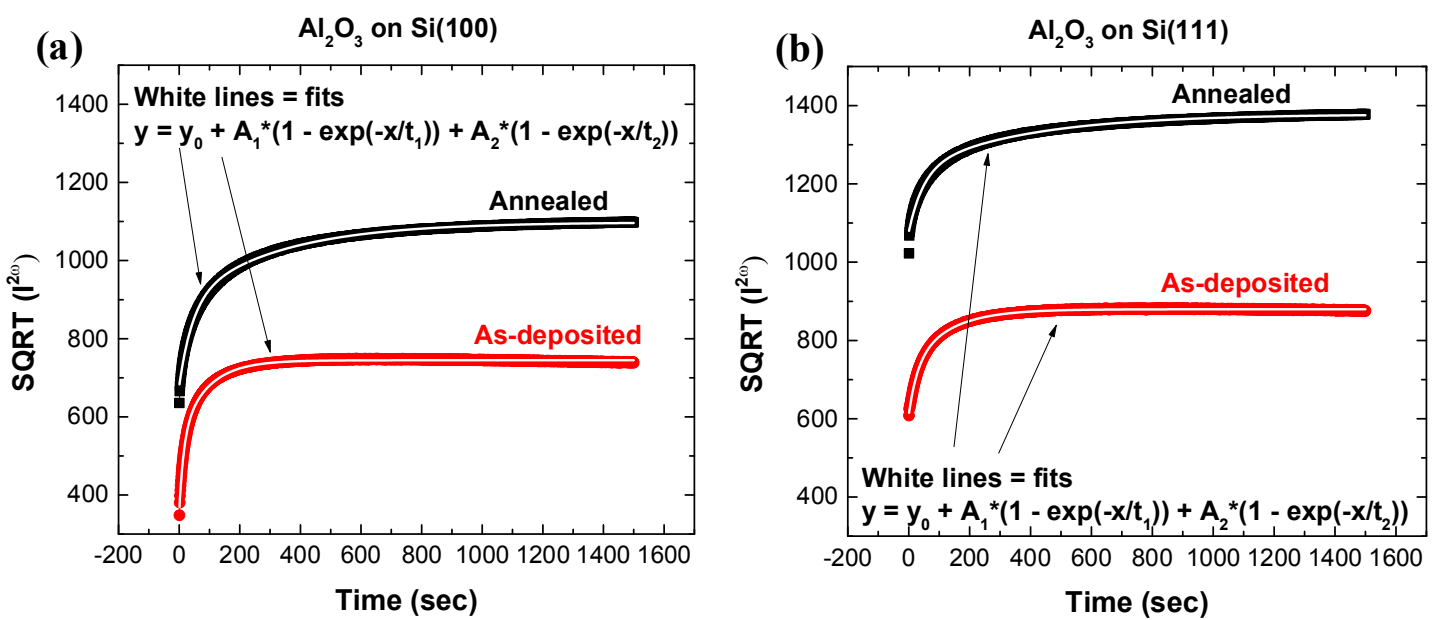

Figure 7: Square-root of SHG intensity versus time for the samples deposited on $\mathrm{Si}(100)$ (a) and on $\mathrm{Si}(111)$ (b). Each graph presents a comparison before and after annealing. The white curves superimposed on the experimental results were obtained by fitting the data with a sum of exponentials.

TABLE I. Time constants from the fits in Figure 7.

\begin{tabular}{ccc}
\hline Sample & $\boldsymbol{\tau}_{\mathbf{1}}(\mathbf{s})$ & $\boldsymbol{\tau}_{\mathbf{2}}(\mathbf{s})$ \\
\hline As-deposited $\mathrm{Al}_{2} \mathrm{O}_{3}$ on $\mathrm{Si}(100)$ & 14.8 & 88.5 \\
Annealed $\mathrm{Al}_{2} \mathrm{O}_{3}$ on $\mathrm{Si}(100)$ & 43.8 & 314.7 \\
As-deposited $\mathrm{Al}_{2} \mathrm{O}_{3}$ on $\mathrm{Si}(111)$ & 27.9 & 123.1 \\
Annealed $\mathrm{Al}_{2} \mathrm{O}_{3}$ on $\mathrm{Si}(111)$ & 48.1 & 367.8 \\
\hline
\end{tabular}




\section{Passivated - Non-passivated SOI Characterization by SHG}

Silicon-on-insulator substrates are highly interesting to enable continued miniaturization in microelectronics, but their characterization before any device fabrication is mandatory. SHG was already used in SOI to evidence charging of the buried oxide due to radiation (28) and the presence of metal contamination on the top of the silicon film (29).

Here we show SHG results of the effect of passivation on SOI wafers. The samples used have $88 \mathrm{~nm}$ thick silicon film and $145 \mathrm{~nm}$ thick buried oxide. One of the samples was passivated with $4 \mathrm{~nm}$ thick thermal oxide, which improves the interface quality by decreasing the interface state density $\left(\mathrm{D}_{\mathrm{it}}\right)$ (30). The SHG results on SOI are correlated with electrical measurements obtained by the pseudo-MOSFET technique.

In the pseudo-MOSFET technique (31), the buried oxide serves as the gate dielectric. The substrate placed on a metallic chuck is biased and used as back-gate. Two metallic probes placed on the top silicon film play the role of source and drain. The current flowing between source and drain is controlled by the voltage applied on the back-gate. The particularity of the pseudo-MOSFET is that channels of electrons and holes can be induced in the same SOI structure, thanks to the metallic source and drain that can provide both electrons and holes in the lightly doped silicon film $\left(\sim 10^{15} \mathrm{~cm}^{-3}\right)$. Consequently, both electrons and holes are tested with the same structure. Figure $8 \mathrm{a}$ shows drain current versus the back-gate voltage on passivated and non-passivated samples. Classical MOSFET methods such as the Y-function (32) allow the extraction of parameters like mobility and threshold voltage. Passivation induced an increase in the mobility, which for the electrons went from $375 \mathrm{~cm}^{2} / \mathrm{V} / \mathrm{s}$ (in the non-passivated sample) to $486 \mathrm{~cm}^{2} / \mathrm{V} / \mathrm{s}$ (in the passivated sample). This is the effect of the decrease of the interface density of states $\mathrm{D}_{\text {it }}$ due to passivation. The effect is also visible in the Figure $8 \mathrm{a}$, in which the sub-threshold slope of the curves is increasing after passivation, indicating reduced $D_{\text {it }}$ values.

The time-dependent SHG signals on these wafers are shown in Figure 8(b). An increased signal is obtained for the non-passivated case, in agreement with a larger $D_{\text {it }}$ value for the non-passivated samples. Note that due to absorption of the SHG by silicon, the signal measured is mainly coming from the top interface for the $88 \mathrm{~nm}$ film thickness. These results confirm SHG can monitor passivation effects and thus interfacial quality.
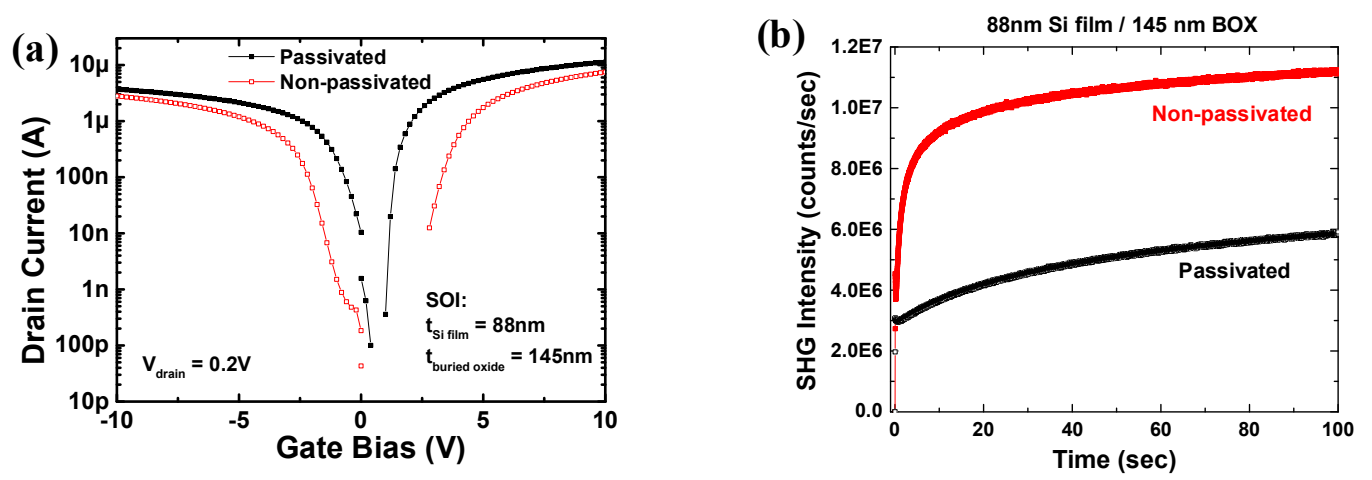

Figure 8: (a) Drain current versus gate voltage in passivated and non-passivated SOI with $88 \mathrm{~nm}$ of Si film thickness and $145 \mathrm{~nm}$ of buried oxide thickness. (b) Time-dependent SHG signal obtained on the passivated and non-passivated samples. 


\section{Conclusions}

In this paper we discuss the use of second harmonic generation to characterize dielectrics on silicon. The technique is contact-less and therefore very appropriate for thin dielectric films. We applied SHG to study $\mathrm{Al}_{2} \mathrm{O}_{3}$ deposited on $\mathrm{Si}(100)$ and $\mathrm{Si}(111)$ substrates. This dielectric layer is known to chemically passivate the silicon surface. A complementary annealing step activates negative charges at the interface, which induces a supplementary field effect passivation. The samples were tested by measuring the carrier lifetime and SHG. The samples with increased lifetime after the annealing are also showing enhanced SHG signal. The SHG signal increase is not related to the lifetime increase but to the stronger electric field induced SHG due to a higher number of charges at the interface after the annealing. The link between SHG and interface charges is confirmed by measurements performed on $\mathrm{SiN}_{\mathrm{x}}$ coated $\mathrm{Si}$ substrates and passivated and non-passivated SOI samples.

As SHG is particularly sensitive to field effect passivation due to charge activation during annealing, it can support technological developments and process monitoring in field effect passivation schemes for photovoltaic cells. Furthermore, SHG provides the unique ability to study charging and discharging dynamics in dielectric / silicon stacks, with no minimum dielectric thickness required since it is contactless.

The time-dependent SHG, which evidenced charging effects due to generated carriers during the experiment, revealed the existence of two charging phenomena/mechanisms in $\mathrm{Al}_{2} \mathrm{O}_{3}$. With the enhanced understanding of the charging effects gained, alongside calibration with electrical methods to accommodate proprietary processing steps, SHG can give non-destructive, fast access to information on charges related to passivation in leading edge and specialized semiconductor device production.

\section{Acknowledgments}

Region Rhone-Alpes (ARC 6 program) is thanked for financial support.

We would also like to thank Vanderbilt University SHG team (Profs. M. Alles, R. Schrimpf, N. Tolk) and SOITEC (O. Kononchuk, F. Allibert) for cooperation.

\section{References}

(1) http://www.itrpv.net/Reports/Downloads/,

(2) http://www.itrs.net/Links/2013ITRS/Home2013.htm,

(3) Gielis, Gevers, Aarts, van de Sanden and Kessels, Journal of Vacuum Science \& Technology A, 26(6), (2008).

(4) Butcher and Cotter, The Elements of Nonlinear Optics, Cambridge University Press, (1991)

(5) Heinz, Second-order nonlinear optical effects at surfaces and interfaces, Elsevier Science, (1991)

(6) Daum, Applied Physics A, 87(3), (2007).

(7) Terlinden, Dingemans, Vandalon, Bosch and Kessels, J App Phys, 115(3), (2014).

(8) Park, Qi, Xu, Varga, Weiss, Rogers, Lupke and Tolk, phys. stat. sol. (b), 247(8), (2010).

(9) Tom, Heinz and Shen, Phys Rev Lett, 51(21), (1983). 
(10) Dadap, Doris, Deng, Downer, Lowell and Diebold, App Phys Lett, 64(16), (1994).

(11) Kotaro, Hideo and Atsuo, Japanese Journal of Applied Physics, 30(5R), (1991).

(12) Li, Willits, Cundiff, Aarts, Stevens and Dessau, App Phys Lett, 89(2), (2006).

(13) Sipe, Moss and Van Driel, Physical Review B, 35(3), (1987).

(14) Gielis, Hoex, van de Sanden and Kessels, J App Phys, 104(7), (2008).

(15) Glinka, Wang, Singh, Marka, Rashkeev, Shirokaya, Albridge, Pantelides, Tolk and Lucovsky, Physical Review B, 65(19), (2002).

(16) Jun, White, Schrimpf, Fleetwood, Brunier, Bresson, Cristoloveanu and Tolk, App Phys Lett, 85(15), (2004).

(17) Lupke, Surface Science Reports, 35(35), (1999).

(18) Fomenko, Gusev and Borguet, J App Phys, 97(8), (2005).

(19) Roll, Mo, Lind, Johansson and Wernersson, App Phys Lett, 106(20), (2015).

(20) Dingemans and Kessels, Journal of Vacuum Science \& Technology A, 30(4), (2012).

(21) Barbos, Blanc-Pelissier, Fave, Blanquet, Crisci and Lemiti, Thin Solid Films,

(22) http://femtometrix.com,

(23) Hoex, Schmidt, Pohl, van de Sanden and Kessels, J App Phys, 104(4), (2008).

(24) Dingemans, Mandoc, Bordihn, van de Sanden and Kessels, App Phys Lett, 98(22), (2011).

(25) Mihaychuk, Shamir and van Driel, Physical Review B, 59(3), (1999).

(26) Mihaychuk, Bloch, Liu and van Driel, Opt. Lett., 20(20), (1995).

(27) Naumann, Otto, Wehrspohn, Werner and Hagendorf, Energy Procedia, 27((2012).

(28) Jun, Schrimpf, Fleetwood, White, Pasternak, Rashkeev, Brunier, Bresson, Fouillat, Cristoloveanu and Tolk, IEEE Transactions on Nuclear Science, 51(6), (2004).

(29) Alles, Pasternak, Lu, Tolk, Schrimpf, Fleetwood, Dolan and Standley, Semiconductor Manufacturing, IEEE Transactions on DO - 10.1109/TSM.2007.896642, 20(2), (2007).

(30) Hamaide, Allibert, Hovel and Cristoloveanu, J App Phys, 101(11), (2007).

(31) Cristoloveanu, Munteanu and Liu, IEEE Transactions on Electron Devices, 47(5), (2000).

(32) Ghibaudo, Electronics Letters, 24(9), (1988). 